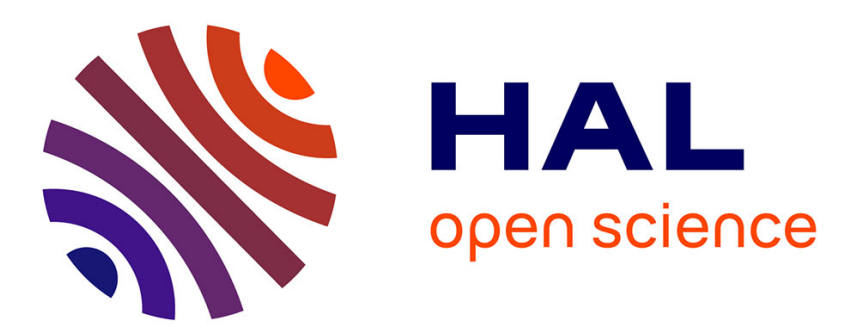

\title{
Application d'un modèle de champ acoustique aléatoire à l'environnement sonore de la maquette Ariane 5
}

\author{
J. Varnier
}

\section{To cite this version:}

J. Varnier. Application d'un modèle de champ acoustique aléatoire à l'environnement sonore de la maquette Ariane 5. Journal de Physique IV Proceedings, 1994, 04 (C5), pp.C5-821-C5-824. 10.1051/jp4:19945177 . jpa-00252858

\section{HAL Id: jpa-00252858 https://hal.science/jpa-00252858}

Submitted on 1 Jan 1994

HAL is a multi-disciplinary open access archive for the deposit and dissemination of scientific research documents, whether they are published or not. The documents may come from teaching and research institutions in France or abroad, or from public or private research centers.
L'archive ouverte pluridisciplinaire $\mathbf{H A L}$, est destinée au dépôt et à la diffusion de documents scientifiques de niveau recherche, publiés ou non, émanant des établissements d'enseignement et de recherche français ou étrangers, des laboratoires publics ou privés. 


\title{
Application d'un modèle de champ acoustique aléatoire à l'environnement sonore de la maquette Ariane 5
}

\section{J. VARNIER}

Office National d'Etudes et de Recherches Aérospatiales, BP. 72, 92322 Châtillon cedex, France

\begin{abstract}
The prediction of the acoustic environment of a launcher at take-off is an important problem to which jet noise theories do not give a satisfactory answer, because of the complex interactions between the jets of the boosters and the structures of the launch pad. Thus, a modelization of the acoustic field has been undertaken directly, using the measurements on the cylinder wall of a captive ARIANE $51 / 20$ scale-model. The measurements were made for several firings of the launcher at various altitudes. The physical model, composed of a limited number of uncorrelated plane waves, takes into account the random character of the incident field and allows us to compute the acoustic field scattered by the wall of the launcher, represented by a portion of an infinite cylinder. The comparisons between computed and measured sound pressure levels and cross-correlation functions allow us to optimize the parameters of the model. This is the first step for determining the acoustic pressure on the payload of the launcher. These computation also give us information on the sound sources and on the efficiency of noise reduction devices.
\end{abstract}

\section{INTRODUCTION}

La prévision de l'ambiance acoustique du lanceur ARIANE 5 au décollage, en vue de déterminer et de limiter les fluctuations de pression auxquelles la charge utile est soumise, fait partie des études menées par l'ONERA sous l'égide du CNES. Les modèles de jet libre issus des travaux de la NASA ne s'appliquent pas ici de façon satisfaisante, en raison des effets complexes d'interaction entre les jets des propulseurs et l'infrastructure au sol.

C'est pourquoi une modélisation expérimentale du champ acoustique a été entreprise directement à partir des mesures effectuées sur la coiffe de la maquette captive ARIANE 5 au 1/20ème, lors des tirs effectués pour le compte de l'Aérospatiale au Centre d'Essais du Fauga-Mauzac, en 1987 et 1992 [1].

Les simulations décrites concernent les très basses altitudes du lanceur ( 0 à $80 \mathrm{~m}$ à l'échelle 1$)$ et une plage de fréquences incluant $90 \%$ de la puissance acoustique émise.

\section{DESCRIPTION DU MODELE}

\subsection{Objectifs et contraintes}

Le champ acoustique incident au niveau de la coiffe du lanceur (partie située au-dessus des propulseurs latéraux, figure 1) doit être modélisé d'une façon simple, mais rendant compte de ses aspects quantitatifs 
(niveaux de pression acoustique mesurés sur la paroi) et qualitatifs (densités spectrales croisées expérimentales entre capteurs proches, renseignant sur sa cohérence et sa direction).

Le modèle doit, en particulier, tenir compte des effets de diffraction par la paroi du lanceur et du caractère aléatoire des sources acoustiques réelles.

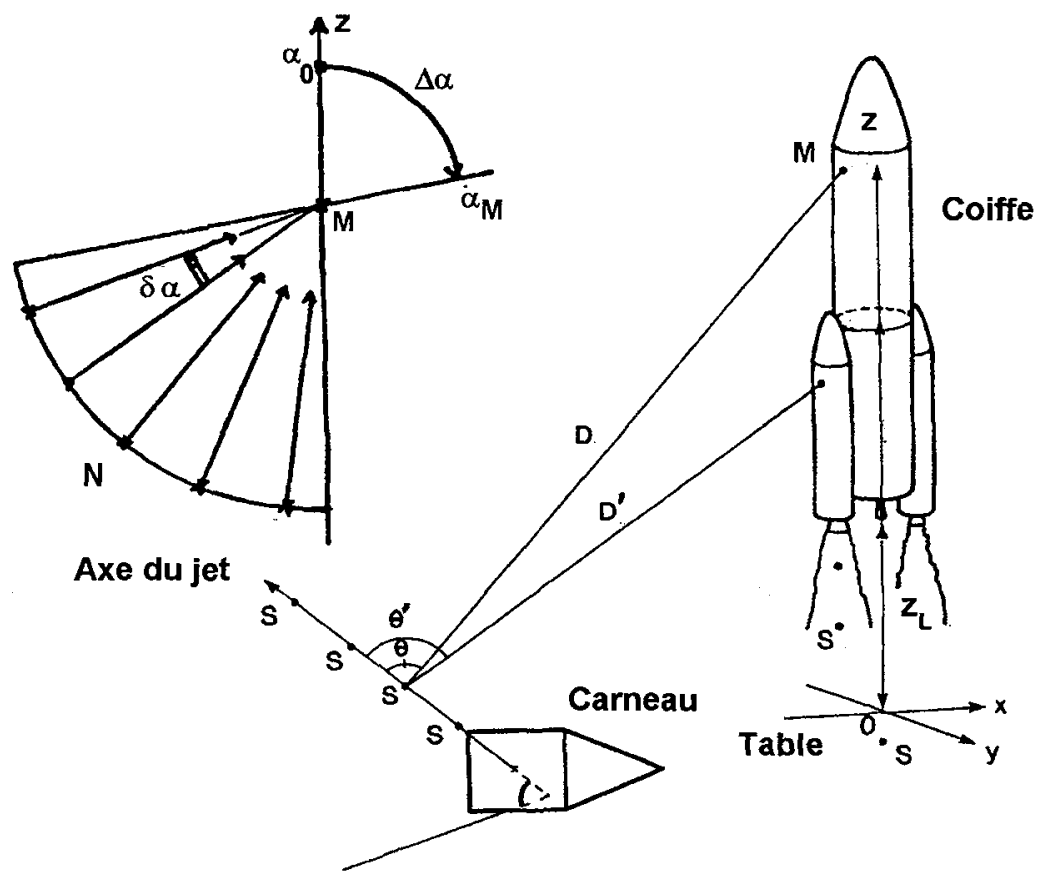

Figure 1 - Modélisation par sources discrètes et modélisation par ondes planes.

\subsection{Solution adoptée}

La modélisation par sources ponctuelles directives $\mathrm{S}$, dérivée de celle du jet libre (figure 1), se heurte notamment à l'éclatement des jets à la sortie des carneaux (tunnels cache-flammes) et à l'effet de jet de sol qui apparaît dès que le lanceur s'élève (divergence des gaz autour du point d'impact).

On adopte donc une démarche inverse basée sur les considérations suivantes : pour un point d'écoute $M$ situé sur le lanceur, le champ incident est constitué d'une distribution continue d'ondes planes incidentes ; celle-ci est modélisée par un nombre restreint d'ondes planes régulièrement réparties en site et en azimut, de manière à ce que le pas adopté représente l'écart angulaire de corrélation des ondes planes élémentaires. La somme quadratique des valeurs efficaces des amplitudes représente en fait la variance d'un champ acoustique "cohérent" (aléatoire mais non diffus). Cette sommation s'effectue après le calcul analytique du champ diffracté [2], sur la paroi de la coiffe assimilée à une portion de cylindre infini, ou à distance de celle-ci (figure 2 ). 


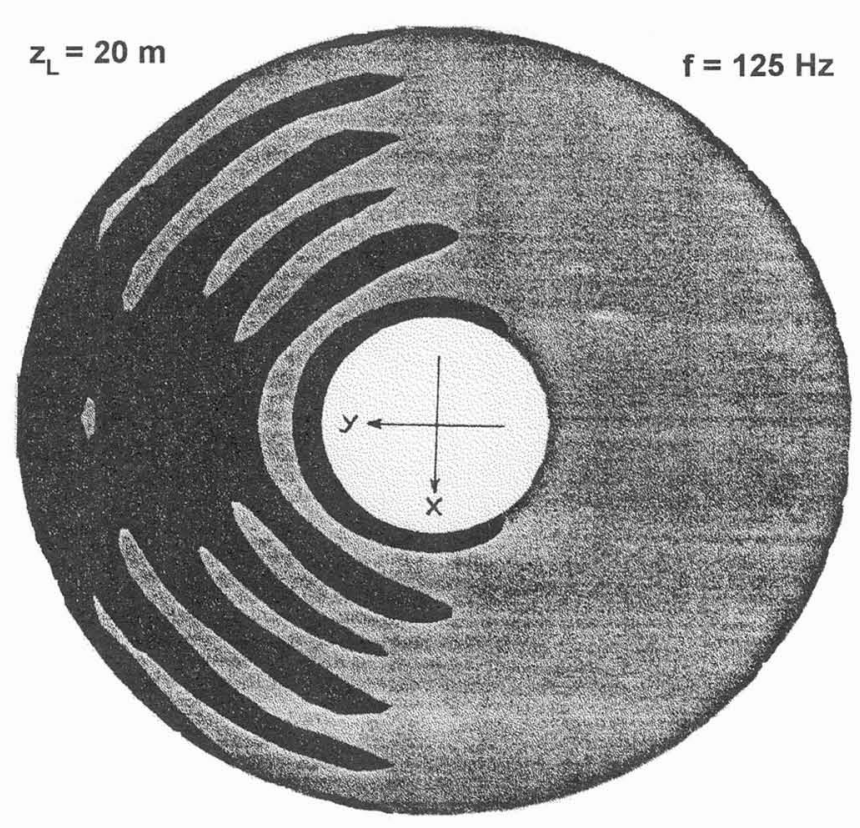

Figure 2 - Carte du champ diffracté environnant la coiffe du lanceur.

\section{SIMULATIONS EFFECTUEES}

\subsection{Densités Spectrales Croisées (DSC)}

Soit deux points de mesure espacés d'une distance verticale h et un champ incident aléatoire diffus limité à un intervalle en site $\Delta \alpha=\alpha_{M}-\alpha_{0}$ (figure 1). On montre que l'expression de la DSC normalisée entre les deux points est :

$$
I_{1,2}=\operatorname{sinc}(\mathrm{k} \cdot \mathrm{h} \cdot \mathrm{d}) \cdot \exp (-\mathrm{j} \cdot \mathrm{k} \cdot \mathrm{h} \cdot \mathrm{s})
$$

avec $\quad d=\left(\cos \alpha_{0}-\cos \alpha_{M}\right) / 2 \quad$ et $\quad s=\left(\cos \alpha_{0}+\cos \alpha_{M}\right) / 2 \quad(k$ nombre d'onde, $j=\sqrt{-1})$. Les changements de signe du sinus cardinal provoquent des sauts de la phase k.h.s. Soit, maintenant, $N$ ondes planes non corrélées et de même amplitude réparties dans l'intervalle $\Delta \alpha$ : les discontinuités théoriques de la phase se traduisent par des irrégularités de pente également constatées dans la phase réelle ; le module calculé est aussi plus proche du module expérimental (figure 3 ).

Cette simulation semble donc confirmer le bien-fondé du modèle adopté. Elle permet de fixer l'ouverture $\Delta \alpha$ et la discrétisation $\mathrm{N}$ en site.

\subsection{Niveaux de pression acoustique}

Les 18 ondes planes du modèle final sont réparties en trois groupes conformément à la configuration des sources réelles présumées (ondes "carneaux", ondes d'ambiance relatives au jet de sol). Une procédure d'optimisation, basée sur la reproduction du "contraste acoustique" mesuré sur la paroi du lanceur (gradient de pression acoustique de la zone éclairée vers la zone d'ombre, cf. figure 2), permet de fixer les amplitudes respectives de ces groupes d'ondes.

Les niveaux de référence sont les niveaux en tiers d'octave moyennés, dans une direction donnée, sur la hauteur de la coiffe. La précision du calcul après lissage en fréquence des amplitudes reste au moins égale à l'incertitude de la mesure ; néanmoins, le modèle est mieux adapté aux fréquences basses et moyennes. 
En effet, on ne tient pas compte du masque que constitue le corps des propulseurs latéraux (figure 1), ni des effets de diffraction qu'ils peuvent induire.
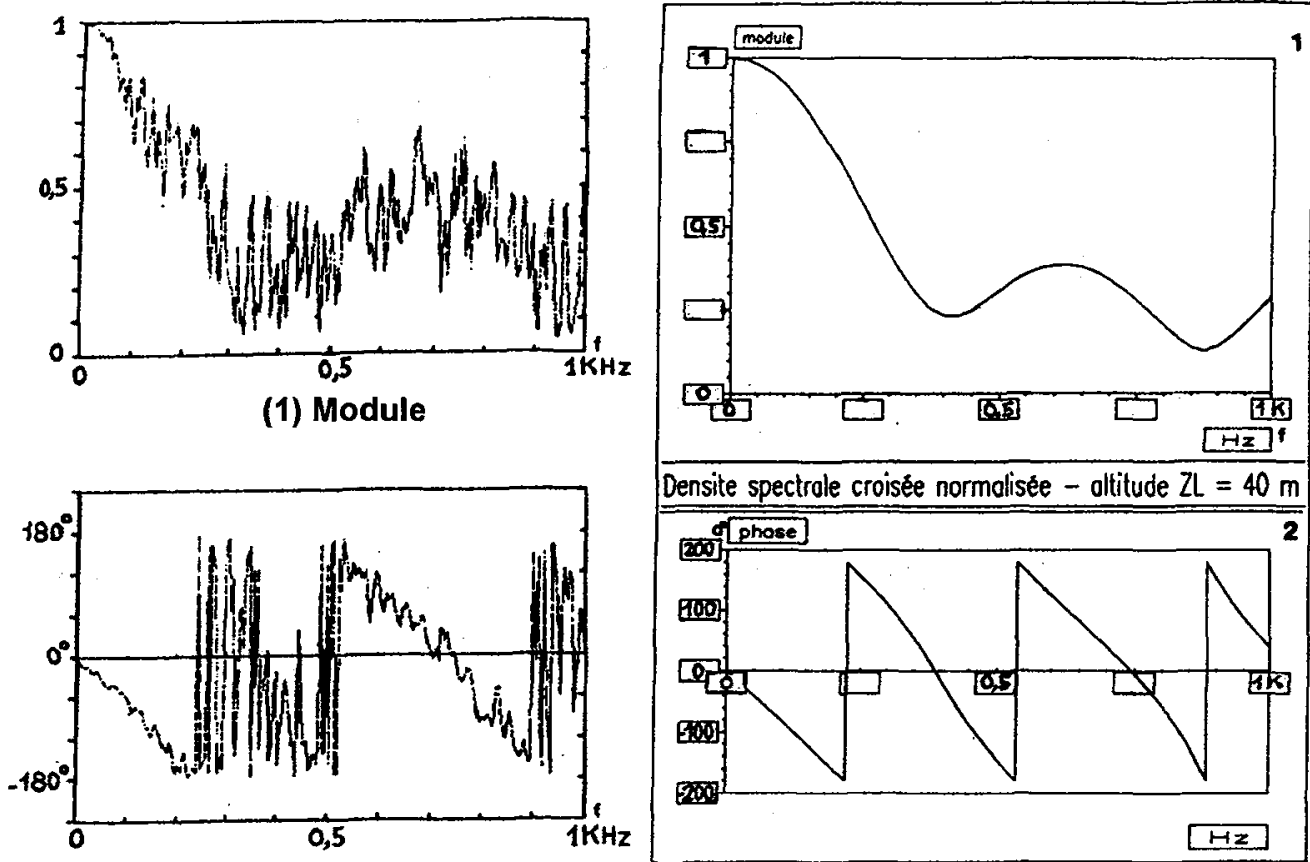

(2) Phase

Figure 3 - Densités spectrales croisées réelle et simulée.

\section{CONCLUSION}

La simulation décrite permet de connaître les paramètres de direction et d'amplitude du jeu d'ondes incidentes, destinés à fournir les entrées d'un code de calcul vibro-acoustique. Elle répond donc au problème posé avec le maximum de fidélité au champ acoustique réel permis par les simplifications effectuées.

L'analyse des résultats fournit en outre, en fonction de l'altitude du lanceur :

* l'évolution du spectre et du niveau global de la pression acoustique incidente,

* des indications concernant l'importance respective des différentes sources de bruit et l'efficacité des dispositifs visant à le réduire.

\section{REFERENCES}

[1] Varnier J., "Diffraction d'un champ acoustique à caractère aléatoire par un cylindre infini. Application à la modélisation de l'environnement acoustique de la maquette ARIANE 5 à partir des données expérimentales", Note Technique ONERA n 1993-11 (Châtillon, 1993).

[2] Waterhouse R.V., "Nearfield diffraction patterns at a sphere or cylinder", J. Acoust. Soc. Am. 75 (1984), 695-706. 